NOTICE: this is the author's version of a work that was accepted for publication in Journal of Process Control. Changes resulting from the publishing process, such as peer review, editing, corrections, structural formatting, and other quality control mechanisms may not be reflected in this document. Changes may have been made to this work since it was submitted for publication. A definitive version was subsequently published in Journal of Process Control, Vol.24, no.7 (2014). DOI: 10.1016/j.jprocont.2014.05.012 


\title{
Generalized Multi-scale Control Scheme for Cascade Processes with Time-Delays
}

Jobrun Nandong ${ }^{*, a}$ and Zhuquan Zang ${ }^{\mathrm{b}}$

${ }^{a}$ Department of Chemical Engineering, Curtin University Sarawak, 98009 Miri, Sarawak, Malaysia.

${ }^{b}$ Department of Electrical and Computer Engineering, Curtin University Sarawak, 98009 Miri, Sarawak, Malaysia.

*Corresponding author: Jobrun Nandong

*Email: jobrun.n@curtin.edu.my; Tel: +6085-443824; Fax:+6085-443838

\begin{abstract}
The cascade control is a well-known technique in process industry to improve regulatory control performance. The use of the conventional PI/PID controllers has often been found to be ineffective for cascade processes with long time-delays. Recent literature report has shown that the multi-scale control (MSC) scheme is capable of providing improved performance over the conventional PID controllers for processes characterized by long time-delays as well as slow RHP zeros. This paper presents an extension of this basic MSC scheme to cascade processes with long time-delays. This new cascade MSC scheme is applicable to self-regulating, integrating and unstable processes. Extensive numerical studies demonstrate the effectiveness of the cascade MSC scheme compared with some well-established cascade control strategies.
\end{abstract}

KEYWORDS: Time-Delay, Cascade Control, Modified Smith Predictor, PID, Multi-Scale Control 


\section{Introduction}

One of the most common control strategies adopted in process industry in order to improve disturbance rejection performance is the cascade control strategy introduced in [1]. Many process control textbooks advocate the benefits of cascade control strategy, i.e., see [2-4]. One of the well-known benefits of cascade control strategy is the ability to correct for certain disturbances in advance before they can seriously influence the primary or main controlled variable.

One well-known example of cascade control application is in reactor temperature control, e.g., polymerization reactor [5]. Here, the cascade control strategy uses the jacket reactor temperature as an extra measurement (secondary output). The role of the secondary controller is to quickly reject any disturbance that initially affects the jacket reactor temperature before the disturbance can seriously affect the primary reactor temperature.

A number of researchers have extensively studied the applications of cascade control scheme to single-input and single-output (SISO) stable processes, e.g., see works by [6-10]. However, much fewer number of researchers have focused on the design of cascade control strategies for unstable or integrating processes with long time-delays. The design of cascade control for these types of processes has been known to be a challenging task due to the presence of unstable modes and delays, which often impose limitation on the achievable control performance. For stable (non-cascade) SISO processes with time-delays, one can improve the regulatory control performance by using the classical Smith predictor [11]. Interestingly, some researchers have also proposed extensions of the classical Smith predictor to SISO non-self-regulating (integrating/unstable) processes, e.g., see works on modified Smith predictors in [12-14]. Additionally, several cascade control strategies based on the Smith predictor have also been developed for non-self-regulating processes with time-delays. Among these cascade control 
strategies based on the Smith predictor are the schemes reported in [15-21]. It should be noted that, the existing modified Smith predictor schemes for the unstable and integrating cascade processes require the design of several controllers. Hence, these Smith predictor-based cascade control systems are rather difficult to design and implement in practice.

In this work, we present a new cascade control strategy constructed based on the SISO multiscale control (MSC) scheme recently reported in Nandong and Zang [22-23]. The key principle of the MSC scheme is to decompose a given plant into a sum of basic modes with distinct speed of responses. It follows that an individual sub-controller is specifically designed to control each of the plant modes. Finally, an overall multi-scale controller is synthesized by combining all of the sub-controllers in such a way that the faster sub-controller is used as a slave to a slower subcontroller; in other words, the sub-controllers are assembled in a cascaded manner. The rationale behind this cascaded combination of all the sub-controllers is to enhance the cooperation among the different plant modes in order to optimize the overall control performance. The works by Nandong and Zang [22-23] have demonstrated that this MSC scheme is able to provide improved nominal performance as well as performance robustness over some well-established control schemes for the nonminimum-phase (NMP) processes. The main novelty of the present work is to extend this SISO MSC scheme to cascade processes which are characterized by long timedelays, where the processes could be stable or integrating or unstable.

The rest of this paper is laid out as follows. In Section 2, some relevant preliminaries are presented. We also describe the basic idea of the multi-scale control (MSC) scheme for a singleinput and single-output (SISO) process. Then in Section 3, we present the extension of this basic MSC scheme to cascade processes as well as a general controller design procedure. Section 4 provides some illustrative examples to demonstrate the effectiveness of the proposed cascade 
MSC scheme as compared to some well-established cascade control schemes. Section 5 finally highlights some concluding remarks and future works.

\section{Preliminaries}

\subsection{Standard Cascade Control Strategy}

Fig. 1 depicts the block diagram of a standard (conventional) cascade control scheme, which consists of a secondary process $P_{2}$ cascaded with a primary process $P_{1}$. Note that, for the cascade control scheme to work effectively, the secondary control-loop must be faster than the primary control-loop. With respect to Fig. 1, the secondary controller $G_{c 2}$ is often referred to as a slave controller while the primary controller $G_{c 1}$ as a master controller. Here, $D_{1}$ and $D_{2}$ represent the input and output disturbance (w.r.t. secondary process) signals respectively.

Based on Fig. 1, the closed-loop transfer function from the master controller output $E$ to the secondary process output $Y_{2}$ is given as

$$
H_{R S}=\frac{Y_{2}}{E}=\frac{G_{c 2} P_{2}}{1+G_{c 2} P_{2}}
$$

Meanwhile, the closed-loop transfer function from the external setpoint $R$ to the primary process output $Y$ can be expressed as follows

$$
H_{R P}=\frac{Y}{R}=\frac{F_{r} G_{c 1} H_{R S} P_{1}}{1+G_{c 1} H_{R S} P_{1}}
$$

where $F_{r}$ denotes the setpoint pre-filter. The setpoint pre-filter is normally a first order transfer function with a unity gain. The filter time constant can be tuned to give a desired setpoint tracking response, e.g., to achieve a desired overshoot for setpoint tracking. 


\subsection{Plant Decomposition}

Consider a rational transfer function $P$ (with numerator $N$ and denominator $D$ ), which can be decomposed via partial fraction expansion into a sum of $n+1$ factors or modes as follows:

$$
P=\frac{N}{D}=P_{0}+P_{1}+P_{2}+\ldots+P_{n}
$$

where $P_{i}, \forall i \in\{0,1,2, \ldots, n\}$ is the plant factor or mode, which is either a first- or second-order

system with real coefficients. The plant factors in (3) are arranged from the slowest factor $P_{0}$ to the fastest $P_{n}$, i.e. the dynamic of $P_{i}$ is slower than that of $P_{i+1}$ for $i=0,1,2, \ldots, n-1$. Here, $P_{0}$ is called the outermost factor and $P_{i}, \forall i \in\{1,2,3 \ldots, n\}$ the inner-layer factor.

\subsection{Deadtime Approximation}

When a given plant model contains a deadtime or time-delay component, the time-delay component is first approximated by a rational transfer function before the plant decomposition is performed as in (3). One of the approximation approaches for the deadtime component is based on the Padé rational approximation [24]:

$$
e^{-\theta s} \approx G_{t d}=\frac{L_{n}(-s)}{L_{n}(s)}
$$

where

$$
L_{n}(s)=\sum_{j=0}^{n}\left(\begin{array}{c}
n \\
j
\end{array}\right) \frac{\theta^{j}(2 n-j) !}{(2 n) !} s^{j}=\sum_{j=0}^{n} \frac{\theta^{j}(2 n-j) ! n !}{(2 n) !(n-j) ! j !} s^{j}
$$

The first-order or 1/1 Padé formulae is often sufficient for many practical applications 


$$
e^{-\theta s} \approx G_{t d}=\left(1-\frac{\theta}{2} s\right) /\left(1+\frac{\theta}{2} s\right)
$$

After approximating the time-delay using either (4) or (6), one can then decompose the approximated plant model as follows

$$
P_{m}=P_{m o} e^{-\theta s} \approx P_{m o} G_{t d}=P_{0}+P_{1}+\ldots+P_{n}
$$

where $P_{m o}$ denotes the delay-free part of the plant model $P_{m}$ and $\theta$ the time-delay.

\subsection{Fundamental of Multi-Scale Control Scheme}

Fig. 2 shows the realization block diagram of a 2-layer multi-scale control (MSC) scheme for a single-input and single-output (SISO) process; see [22-23] for further details. The block diagram shown in Fig. 2 implies that the given plant $P$ can be decomposed into a sum of 2 factors or modes with distinct speeds of responses (time-scales) to a similar input. Here, $K_{0}$ and $K_{1}$ denote the sub-controllers corresponding to the outermost and inner-layer factors, respectively; $W_{1}$ is called the multi-scale predictor.

For the 2-layer MSC scheme (Fig. 2), the multi-scale predictor is chosen as

$$
W_{1}=\bar{P}_{1}
$$

where $\bar{P}_{1}$ denotes the nominal model for the plant factor $P_{1}$. The inner-loop of the MSC scheme (Fig. 2a) can be reduced to a standard single-loop feedback control (Fig. 2b). Based on Fig. 2b, we can write the closed-loop inner-layer transfer function as follows

$$
G_{1}=\frac{K_{1}}{1+K_{1} W_{1}}
$$


The augmented overall plant transfer function is given by

$$
P_{c}=G_{1} P
$$

Hence, the overall closed-loop setpoint transfer function from $R$ to $Y$ can be obtained as

$$
H_{r}=\frac{Y}{R}=\frac{F_{r} K_{0} P_{c}}{1+K_{0} P_{c}}
$$

and the overall closed-loop disturbance transfer function from $D_{2}$ to $Y$ as

$$
H_{d}=\frac{Y}{D_{2}}=\frac{P_{1}}{1+K_{0} P_{c}}
$$

Notice that in (11) and (12), the time-delay component remains embedded in the characteristic equations (feedback loop) even at the nominal condition. Hence, this indicates that there is no cancellation of the time-delay component as in the case of the deadtime compensator approach, e.g., the Smith predictor [11].

\section{The Proposed Cascade Multi-Scale Control Scheme}

\subsection{Extension of the MSC Scheme to Cascade Processes}

Fig. 3 illustrates the realization block diagram of a two-level Cascade Multi-Scale Control (CMSC) scheme. For the proposed CMSC scheme, each secondary and primary process has only one inner-layer factor and one outermost factor, i.e., 2-layer MSC scheme for each secondary and primary process; please note that, many real processes can be sufficiently approximated by two basic modes only.

With respect to the secondary process $P_{2}$, the secondary sub-controllers $\mathcal{K}_{s} \in R^{2}$ 


$$
\mathcal{K}_{s}=\left[\begin{array}{c}
K_{s 0} \\
K_{s 1}
\end{array}\right]
$$

As for the primary process $P_{1}$, the primary sub-controllers $\mathcal{K}_{p} \in R^{2}$

$$
\mathcal{K}_{\boldsymbol{p}}=\left[\begin{array}{c}
K_{p 0} \\
K_{p 1}
\end{array}\right]
$$

We can write the closed-loop inner-layer transfer function for the secondary process as

$$
G_{s 1}=\frac{U}{C_{s 0}}=\frac{K_{s 1}}{1+K_{s 1} W_{s 1}}
$$

where $W_{s 1}$ denotes the multi-scale predictor for the secondary process (control system).

The augmented overall secondary plant transfer function can be expressed as

$$
P_{s o}=\frac{Y_{2}}{C_{s 0}}=G_{s 1} P_{2}
$$

Subsequently, the overall closed-loop transfer function from $C_{p 1}$ to $Y_{2}$ is obtained as

$$
H_{R S}=\frac{Y_{2}}{C_{p 1}}=\frac{K_{s 0} P_{s o}}{1+K_{s 0} P_{s o}}
$$

The closed-loop inner-layer transfer function for the primary process is

$$
G_{p 1}=\frac{K_{p 1} H_{R S}}{1+K_{p 1} W_{p 1} H_{R S}}
$$

where $W_{p 1}$ denotes the multi-scale predictor for the primary process.

The augmented overall primary plant transfer function is written as 


$$
P_{p o}=\frac{Y}{C_{p 0}}=G_{p 1} P_{1}
$$

Note that, the overall closed-loop transfer function from $R$ to $Y$ is

$$
H_{R P}=\frac{Y}{R}=\frac{F_{r} K_{p 0} P_{p o}}{1+K_{p 0} P_{p o}}
$$

\subsection{Generalized Cascade Multi-scale Control Scheme}

Consider a generalized cascade MSC (CMSC) system where the secondary and primary processes can be decomposed into $n+1$ and $m+1$ sums of basic factors, respectively:

$$
\left[\begin{array}{l}
P_{1} \\
P_{2}
\end{array}\right]=\left[\begin{array}{c}
P_{p 0}+P_{p 1}+P_{p 2}+\cdots+P_{p m} \\
P_{s 0}+P_{s 1}+P_{s 2}+\cdots+P_{s n}
\end{array}\right]
$$

where the secondary and primary multi-scale predictors are chosen as $W_{s i}=\bar{P}_{s i}, \forall i \in\{1,2, \ldots, n\}$ and $W_{p i}=\bar{P}_{p i}, \forall i \in\{1,2, \ldots, m\}$, respectively. The generalized CMSC scheme is shown in Fig. 4. Referring to Fig. $4 \mathrm{~b}, K_{T}=K_{s 0} G_{s 1} G_{s 2} \cdots G_{s n}$ denotes the overall (master) multi-scale controller corresponding to the secondary process $P_{2}$ where $G_{s i}, \forall i \in\{1,2, \ldots, n\}$ is given by a vector of closed-loop inner-layer transfer functions $\boldsymbol{G}_{s} \in R^{n}$

$$
\boldsymbol{G}_{\boldsymbol{s}}=\left[\begin{array}{c}
G_{s 1} \\
G_{s 2} \\
\vdots \\
G_{s n-1} \\
G_{s n}
\end{array}\right]=\left[\begin{array}{c}
\frac{K_{s 1}}{1+K_{s 1} W_{s 1}\left(\prod_{j=2}^{n} G_{s j}\right)} \\
\frac{K_{s 2}}{1+K_{s 2} W_{s 2}\left(\prod_{j=3}^{n} G_{s j}\right)} \\
\vdots \\
\frac{K_{s n-1}}{1+K_{s n-1} W_{s n-1}\left(\prod_{j=n}^{n} G_{s j}\right)} \\
\frac{K_{s n}}{1+K_{s n} W_{s n}}
\end{array}\right] ; \quad n>1
$$

Next, we also construct a vector of augmented inner-layer transfer functions $\mathcal{Q}_{s} \in R^{n-1}$ 


$$
\mathcal{Q}_{\boldsymbol{s}}=\left[\begin{array}{c}
Q_{s 1} \\
Q_{s 2} \\
\vdots \\
Q_{s n-2} \\
Q_{s n-1}
\end{array}\right]=\left[\begin{array}{c}
\prod_{j=2}^{n} G_{s j} W_{s 1} \\
\prod_{j=3}^{n} G_{s j} W_{s 2} \\
\vdots \\
\prod_{j=n-1}^{n} G_{s j} W_{s n-2} \\
\prod_{j=n}^{n} G_{s j} W_{s n-1}
\end{array}\right] ; n>1
$$

It is important to note that, $\mathcal{Q}_{s}$ is useful for the synthesis of $K_{s i}, \forall i \in\{1,2,3, \ldots, n\}$ as will be shown in the next Section 3.3.

We can write the generalized augmented overall secondary plant transfer function as follows

$$
P_{s o}=\prod_{j=1}^{n}\left(G_{s j}\right) P_{2}
$$

Meanwhile, the overall closed-loop transfer function for the secondary process is given by

$$
H_{R S}=\frac{Y_{2}}{C_{p m}}=\frac{K_{s 0} P_{s o}}{1+K_{s 0} P_{s o}}
$$

To obtain the overall (slave) MSC controller for the secondary process, use

$$
G_{c 2}=K_{s 0}\left(\prod_{j=1}^{n} G_{s j}\right)
$$

It should be noted that the MSC controller (26) can be easily rearranged into an equivalent PID controller augmented with a filter; see [23].

Based on Fig. 4b, a vector of closed-loop (primary) inner-layer transfer functions $G_{p} \in R^{m}$ is expressed as 


$$
\boldsymbol{G}_{\boldsymbol{p}}=\left[\begin{array}{c}
G_{p 1} \\
G_{p 2} \\
\vdots \\
G_{p m-1} \\
G_{p m}
\end{array}\right]=\left[\begin{array}{c}
\frac{K_{p 1} H_{R S}}{1+K_{p 1} W_{p 1} H_{R S}\left\{\prod_{j=2}^{m} G_{p j}\right\}} \\
\frac{K_{p 2} H_{R S}}{1+K_{p 2} W_{p 2} H_{R S}\left\{\prod_{j=3}^{m} G_{p j}\right\}} \\
\vdots \\
\frac{K_{p m-1} H_{R S}}{1+K_{p m-1} W_{p m-1} H_{R S}\left\{\prod_{j=m}^{m} G_{p j}\right\}} \\
\frac{K_{p m} H_{R S}}{1+K_{p m} H_{R S} W_{p m}}
\end{array}\right] ; \quad m>1
$$

Subsequently, a vector of augmented (primary) inner-layer transfer function $\mathcal{Q}_{\boldsymbol{p}} \in R^{m-1}$ is constructed as follows

$$
\mathcal{Q}_{\boldsymbol{p}}=\left[\begin{array}{c}
Q_{p 1} \\
Q_{p 2} \\
\vdots \\
Q_{p m-2} \\
Q_{p m-1}
\end{array}\right]=\left[\begin{array}{c}
\prod_{j=2}^{m} G_{p j} W_{p 1} \\
\prod_{j=3}^{m} G_{p j} W_{p 2} \\
\vdots \\
\prod_{j=m-1}^{m} G_{p j} W_{p m-2} \\
\prod_{j=m}^{m} G_{p j} W_{p m-1}
\end{array}\right] ; \quad m>1
$$

where the augmented overall primary plant transfer function can be written as

$$
P_{p o}=\frac{Y}{C_{p 0}}=\prod_{j=1}^{m}\left(G_{p j}\right) P_{1}
$$

From (29), we can obtain the overall closed-loop transfer function from $R$ to $Y$ as follows

$$
H_{R P}=\frac{F_{r} K_{p 0} P_{p o}}{1+K_{p 0} P_{p o}}
$$

Note that, the overall master MSC controller for the primary process can generally be expressed as 


$$
G_{c 1}=K_{p 0} F
$$

where the augmented filter takes the form of

$$
F=\frac{K_{p 0}\left(\prod_{i=1}^{m} K_{p i}\right)}{1+\sum_{i=1}^{m}\left[\prod_{j=i}^{m}\left(K_{p j} W_{p j}\right)\right]}
$$

For a case where the primary process is only decomposable into 2 basic modes, the filter is

$$
F=\frac{K_{p 1}}{1+K_{p 1} W_{p 1} H_{R S}}
$$

It should be noted that, the overall MSC controller (31) can be reduced to an equivalent PID controller augmented with a filter. When the filter (32) and (33) takes a high-order form, we can reduce the order of the filter using a model order reduction technique, e.g., available in the Matlab Control System Toolbox.

\subsection{Controller Synthesis for the CMSC Scheme}

We propose a general procedure to synthesize CMSC system for a two-level cascade process.

Step 1: Assuming that both primary and secondary processes are with deadtimes, apply the 1/1 Padé formula to first approximate the deadtime components by rational transfer functions. Then, use partial fraction expansion to decompose the approximated primary and secondary plant models into a sum of $m+1$ and $n+1$ basic modes, respectively.

Step 2: Design the sub-controllers for the secondary process assuming that the secondary multiscale predictors are selected as $W_{s i}=\bar{P}_{s i}, \forall i \in\{1,2, \ldots, n\}$.

Step 2.1: Design the innermost sub-controller $K_{s n}$ first based on $W_{s n}=\bar{P}_{s n}$.

Step 2.2: Derive $Q_{s n-1}$ as in (23), and then design the sub-controller $K_{s n-1}$ based on $Q_{s n-1}$. 
Step 2.3: Derive $Q_{s n-2}$ as in (23), which is then used to design $K_{s n-2}$. Repeat this step to design the remaining (secondary) inner-layer sub-controllers $\left(K_{s n-3}, K_{s n-4}, \ldots, K_{s 1}\right)$.

Step 2.4: Derive the augmented overall secondary plant transfer function $P_{s o}$ as in (24). Then, design the secondary outermost sub-controller $K_{s 0}$ based on $P_{s o}$. The overall PID controller can be obtained as in [23].

Step 3: Design the sub-controllers for the primary process assuming that the primary multi-scale predictors are chosen as $W_{p i}=\bar{P}_{p i}, \forall i \in\{1,2, \ldots, m\}$.

Step 3.1: Construct the transfer function $G_{p m}$ as in (27). Based on $G_{p m}$, design the innermost sub-controller $K_{p m}$.

Step 3.2: Construct the transfer function $Q_{p m-1}$ as in (28), and use $Q_{p m-1}$ to synthesize $K_{p m-1}$. Repeat this step to synthesize $K_{p m-2}, K_{p m-3}, \ldots, K_{p 1}$.

Step 3.3: Develop the augmented overall plant transfer function $P_{p o}$ as in (29). Finally, the primary outermost sub-controller $K_{p 0}$ is synthesized based on $P_{p o}$. The overall MSC controller is obtained using (31). This overall controller can then be rearranged in the form of a PID controller augmented with a filter.

Remark: For the inner-layer sub-controllers (either primary or secondary process), it is recommended to use a simple control law, e.g., a proportional (P) controller because it only requires simple tuning and thus, simplifying the overall multi-scale controller synthesis. The outermost sub-controller for the secondary process can also be chosen as a $\mathrm{P}$ controller but if a desired performance cannot be achieved, then one can try to use a more complex control algorithm, e.g., proportional-integral (PI) controller. As for the outermost sub-controller used in 
the primary control-loop (process), it is recommended to use at least a PI controller to remove the steady-state offset. If a desired performance cannot be met, then one can try to use a more complex control algorithm, e.g., a PID with a lag filter or an LQG controller. The proposed design procedure for the CMSC scheme can be easily implemented with the aid of Matlab SISO Design Tool available from the Matlab Control System Toolbox. In this work, the Matlab SISO Design Tool is adopted to synthesize the required controllers i.e., automated controller tuning is applied. Note that, we use the tuning procedure based on the minimum Integral Absolute Error (IAE) criterion for the P/PI/PID controller design.

\section{Illustrative Examples}

The applicability and effectiveness of the proposed CMSC scheme are demonstrated using 3 examples. Please note that, all the P/PI/PID controllers used in the proposed CMSC and standard cascade PID control schemes are designed via the Matlab SISO Design Tool where the tuning is based on the minimum IAE criterion. The simulation is carried out using Matlab Simulink where the stiff ode15ssolver is adopted due to the multi-scale dynamics of the systems involved.

Example 1: Assume the following two stable cascade processes given by

$$
\begin{aligned}
& P_{1}=\frac{\left(1+\Delta_{1}\right) e^{-5\left(1+\delta_{1}\right) s}}{28 s^{2}+1.1 s+1} \\
& P_{2}=\frac{\left(1+\Delta_{2}\right) e^{-4\left(1+\delta_{2}\right) s}}{5 s+1}
\end{aligned}
$$

In equations (34) and (35), $\Delta_{i}$ and $\delta_{i}$ represent modeling errors for the gain and time-delay (TD) of a given process $P_{i}$ respectively. 
CMSC scheme: The multi-scale controllers are synthesized using the design procedure given in Sub-Section 3.3 and with the aid of Matlab SISO Design Tool. Upon approximating the delays in (34) and (35) using the 1/1 Padé formula, we then perform the decomposition which yields:

$$
\begin{aligned}
& {\left[\begin{array}{c}
\bar{P}_{p 0} \\
\bar{P}_{p 1}
\end{array}\right]=\left[\begin{array}{c}
\frac{-5.234 s+0.533}{28 s^{2}+3 s+1} \\
\frac{0.467}{2.5 s+1}
\end{array}\right]} \\
& {\left[\begin{array}{c}
\bar{P}_{s 0} \\
\bar{P}_{s 1}
\end{array}\right]=\left[\begin{array}{c}
\frac{2.335}{5 s+1} \\
\frac{-1.334}{2 s+1}
\end{array}\right]}
\end{aligned}
$$

The multi-scale predictors for the primary and secondary processes are chosen as $W_{p 1}=\bar{P}_{p 1}$ and $W_{s 1}=\bar{P}_{s 1}$, respectively. Here, $\bar{P}$ denotes the nominal model for $P$. For the primary process, the outermost sub-controller $K_{p 0}$ is chosen as a PID controller. All other sub-controllers are chosen as P-only controllers. The following sub-controllers are obtained: $K_{s 0}=-2.1$ and $K_{s 1}=-2.6$ for the secondary process; $K_{p 0}=0.09\left(6 s^{2}+1.1 s+1\right) / s$ and $K_{p 1}=1.1$ for the primary process. All of the sub-controllers are tuned based on the minimum IAE criterion (in Matlab SISO Design Tool). The equivalent PID controller settings corresponding to the multiscale sub-controllers are shown in Table 4. Notice that for the primary process, the master MSC controller is equivalent to a PID controller augmented with a second-order filter, while for the secondary process the slave MSC controller is the same as a P-only controller augmented with a lead-lag filter. Therefore, the proposed CMSC scheme is not much more complex than the standard cascade PID control; the only major difference between the two schemes lies is the augmented filter. Here, the proposed CMSC structure provides an effective way to design the augmented filter for a PID controller. 
Standard Cascade PID scheme: For the standard cascade PID control as shown in Fig. 1, the controllers are designed as follows. The slave controller $G_{c 2}$ is designed first and followed by the master controller $G_{c 1}$. A PI controller is selected for $G_{c 2}$ while a PID controller with a lag filter is chosen for $G_{c 1}$. The slave controller $G_{c 2}$ is designed based on the nominal model of the secondary process. Subsequently, the master controller $G_{c 1}$ is designed based on $H_{R S} \bar{P}_{1}$, where $H_{R S}$ is as in (17) and $\bar{P}_{1}$ is the nominal model for primary process $P_{1}$. Note that, all the delay components appearing in the characteristic equations (17) and (20) are approximated first using the second-order Padé formula. In this case, we use a higher order approximation of the delay in order to perform more accurate Nyquist stability margin analysis. Please note that, for the purpose of CMSC design, the first order approximation of the delay is quite sufficient; however, a higher order approximation will be used if the delay is very long, i.e., larger than the dominant time-constant of the process involved. The Matlab SISO Design Tool is used to obtain the PID controllers based on the minimization of IAE tuning. We obtain a PI (slave) and PID (master) controllers as displayed in Table 4.

For this example, the same set point pre-filter $F_{r}=1 /(0.1 s+1)$ is used for the proposed CMSC and the standard cascade PID control schemes. The performances of the 2 different control schemes are compared based on 1 unit step change in $R$ at $\mathrm{t}=5$, subsequently followed by -0.5 units step change in the input disturbance $D_{1}$ at $\mathrm{t}=200$, and then by -0.5 units step change in the output disturbance $D_{2}$ at $\mathrm{t}=400$. To compare the performance robustness of the 2 control schemes, the following 4 perturbed conditions are considered:

i. Case A: $\Delta_{1}=\Delta_{2}=0.2$ and $\delta_{1}=\delta_{2}=0.2$ (simultaneous increase in the gain and TD modeling errors of magnitude $+20 \%$ each). 
ii. Case B: $\Delta_{1}=0.2, \Delta_{2}=-0.2$ and $\delta_{1}=\delta_{2}=0.2 \quad(20 \%$ increases in the primary and decreases in the secondary process gains with $20 \%$ delay errors in both primary and secondary).

iii. Case C: $\Delta_{1}=-0.2, \Delta_{2}=0.2$ and $\delta_{1}=\delta_{2}=0.2(20 \%$ decreases in the primary and $20 \%$ increases in the secondary process gains with $20 \%$ delay errors).

iv. Case D: $\Delta_{1}=-0.2, \Delta_{2}=-0.2$ and $\delta_{1}=\delta_{2}=0.2$ (decrease in both the primary and secondary process gains with $20 \%$ delay errors).

For all of the cases mentioned above, we are concerned with the effects of positive time-delay (TD) modeling errors ( $\left.\delta_{i}>0, i=1,2\right)$ and not with the negative errors $\left(\delta_{i}<0, i=1,2\right)$ because the simultaneous increases in time-delays (positive modeling errors) tend to cause more serious degradation in control performance for a given set of gain modeling errors.

The IAE values for the 2 control schemes at the nominal and perturbed conditions are as displayed in Table 1. The tabulated IAE values indicate that the proposed CMSC scheme outperforms the standard cascade PID scheme in terms of both nominal performance and performance robustness. On average, the CMSC provides about $30 \%$ improvement over the cascade PID in term of IAE. Fig. 5 shows the comparative closed-loop responses under a perturbed condition for the 2 different cascade control strategies. The proposed CMSC scheme shows improved setpoint tracking as well as regulatory control performances over the standard cascade scheme. Fig. 6 shows the plots of gain margin versus time-delay errors for two sets of gain modeling errors. From Fig. 6, notice that the CMSC scheme has a larger gain/delay margin than the standard cascade PID scheme, which implies that the CMSC is more robust than the standard cascade PID scheme. This confirms the simulation result shown in Fig. 5, i.e., the CMSC has better performance robustness than the standard cascade PID control scheme. Please 
note that, the gain margins of both CMSC and standard cascade PID schemes are virtually similar at the nominal condition (see Fig. 6b). Interestingly, despite the similarity in gain margins at the nominal condition, the CMSC exhibits better performance (in term of IAE) than the cascade PID scheme (see Table 1).

Example 2: In this example, we consider the double integrating primary process reported in Uma et al. [17]:

$$
\begin{aligned}
& P_{1}=\frac{\left(1+\Delta_{1}\right) e^{-0.7\left(1+\delta_{1}\right) s}}{s^{2}} \\
& P_{2}=\frac{\left(1+\Delta_{2}\right) e^{-0.3\left(1+\delta_{2}\right) s}}{s+1}
\end{aligned}
$$

For the example, we compare the performances of 3 different control schemes: (1) the proposed CMSC, (2) standard cascade PID control, and (3) modified Smith predictor (SP) of Uma et al. [17].

CMSC scheme: First, we apply the 1/1 Padé formula to approximate the delay components in the primary and secondary process models (42) and (43). Then, the decompositions of the approximated models result in the following factors:

$$
\begin{aligned}
& {\left[\begin{array}{c}
\bar{P}_{p 0} \\
\bar{P}_{p 1}
\end{array}\right]=\left[\begin{array}{c}
\frac{-0.7 s+1}{s^{2}} \\
\frac{-0.7}{s+2.857}
\end{array}\right]} \\
& {\left[\begin{array}{l}
\bar{P}_{s 0} \\
\bar{P}_{s 1}
\end{array}\right]=\left[\begin{array}{c}
\frac{1.3529}{s+1} \\
\frac{-0.3529}{0.15 s+1}
\end{array}\right]}
\end{aligned}
$$

We choose the outermost sub-controller $K_{p 0}$ as a PID controller while the other 3 subcontrollers are selected as P-only controllers. Using the design procedure given in Sub-Section 
3.3, the following sub-controllers are obtained: $K_{p 1}=1.1$ and $K_{p 0}=0.045\left(21.7 s^{2}+8.3 s+1\right) / s$ for the primary process; $K_{s 1}=-3$ and $K_{s 0}=-1.8$ for the secondary process. These multi-scale sub-controllers can be reduced to an equivalent PID augmented with a second order filter, and a $\mathrm{P}$ controller with a lead-lag filter as displayed in Table 4.

Standard Cascade PID: For a comparison purpose, we also design a standard cascade PID control scheme. We choose a PI controller for controlling the secondary process and a PID controller for the primary process. These PI and PID controller designs are performed just like in the previous Example 1. The controller tuning is based on the minimum IAE criterion. The PI and PID controllers obtained are given in Table 4.

Note that, the same setpoint prefilter $F_{r}=1 /(4 s+1)$ is used for both CMSC and standard cascade PID control schemes.

Modified SP: For another comparison, we also adopt the modified Smith predictor (SP) scheme proposed by Uma et al. [17]. Please note that, all the controllers for the modified SP scheme use in this example are exactly as reported in [17], i.e., no modification. For details regarding the controller design for the modified SP, refer to [17]. The 4 controllers used in the modified SP scheme are shown in Table 4. Notice that the modified SP scheme is a more complex design than the proposed CMSC scheme. It is interesting to note that unlike the proposed CMSC scheme, the modified SP scheme cannot be reduced to an equivalent standard cascade control scheme.

The control performances are compared based on 1 unit step change in $R$ at $\mathrm{t}=5$ units, and consecutively followed by -0.5 units step change each in $D_{1}$ at $\mathrm{t}=75$ units and $D_{2}$ at $\mathrm{t}=150$ units. To compare the closed-loop performances in the presence of modeling errors, 4 cases of perturbed conditions similar to those described in the previous Example 1 are considered. 
Table 2 displays the IAE values of the different control schemes at the nominal and perturbed conditions. The table demonstrates that the proposed CMSC scheme outperforms the standard cascade and modified SP schemes in terms of the nominal performance and robust performance. On average, the CMSC scheme provides about $30 \%$ and $35 \%$ performance improvement over the standard cascade PID and modified SP schemes. Fig. 7 shows the closed-loop responses at a perturbed condition for the 3 different control schemes. The figure indicates that the CMSC scheme yields good setpoint tracking and regulatory control performances compared with the other 2 schemes. Fig. 8 illustrates the gain margins versus delay errors of the CMSC and standard cascade scheme; the modified SP is not shown as it is not equivalent to a standard cascade control strategy. It is interesting to highlight that, although the primary-loop delay margins (delay error at which the control scheme becomes unstable) for both CMSC and standard cascade control are similar, the CMSC exhibits higher gain margin than the standard cascade control. This accounts for a greater performance robustness of the CMSC than the standard cascade control.

Example 3: This example is adopted from Uma et al. [17] where the primary process is an open-loop unstable. The cascade processes are given as follows:

$$
\begin{aligned}
& P_{p}=\frac{\left(1+\Delta_{1}\right) e^{-4\left(1+\delta_{1}\right) s}}{20 s-1} \\
& P_{s}=\frac{\left(1+\Delta_{2}\right) 2 e^{-2\left(1+\delta_{2}\right) s}}{20 s+1}
\end{aligned}
$$

Note that, Uma et al. [17] reported that their cascade control strategy based on a modified SP can provide improved performance over the control schemes proposed by [20-21]. It is interesting to find out whether the proposed CMSC scheme can provide further performance improvement over the modified SP scheme proposed in [17]. 
CMSC scheme: After the rational approximation of the delays in (50) and (51), the plant decomposition of the approximated models leads to:

$$
\begin{aligned}
& {\left[\begin{array}{l}
\bar{P}_{p 0} \\
\bar{P}_{p 1}
\end{array}\right]=\left[\begin{array}{c}
\frac{-0.95}{20 s-1} \\
\frac{0.098}{2 s+1}
\end{array}\right]} \\
& {\left[\begin{array}{l}
\bar{P}_{s 0} \\
\bar{P}_{s 1}
\end{array}\right]=\left[\begin{array}{c}
\frac{2.21}{20 s+1} \\
\frac{-0.21}{s+1}
\end{array}\right]}
\end{aligned}
$$

Based on the procedure given in Sub-Section 3.3, 4 sub-controllers are produced as: $K_{p 1}=4.2$ and $K_{p 0}=0.034\left(39 s^{2}+30 s+1\right) / s$ for the primary process; $K_{s 1}=-40$ and -0.95 for the secondary process. The equivalent P (slave) and PID (master) controllers are shown in Table 4.

Standard Cascade PID: A PI and PID controllers are chosen for controlling the secondary and primary processes, respectively. We use the same controller design procedure as in the previous 2 examples. The result of the controller design is given in Table 4.

Here, the same setpoint prefilter $F_{r}=1 /(28 s+1)$ is used for both CMSC and standard cascade PID control schemes.

Modified SP: Since this example is directly taken from Uma et al. [17], the modified SP used in this study is exactly the same as in the original paper. For comparison with the other 2 schemes, the controllers used in the modified SP are as displayed in Table 4. Bear in mind that, the modified SP structure is not reducible to an equivalent standard cascade control as the proposed CMSC scheme. In term of design and structure, the modified SP are more complex than the proposed CMSC scheme.

The performances of the 3 different control schemes are compared based on 1 unit step change in $R$ at $\mathrm{t}=5$ units, and consecutively followed by 2 units step change each in $D_{1}$ and $D_{2}$ at $\mathrm{t}=$ 
150 units and $t=300$ units, respectively. The performance robustness is evaluated at a set of perturbed conditions similar to those used in the Examples 1 and 2.

Table 3 reveals the IAE values corresponding to the 3 different control schemes. As can be seen from the table, just like in the previous examples the CMSC outperforms the other 2 control schemes at all perturbed conditions as well as at the nominal condition. For the cascade PID control, we can further improve the control performance at the nominal condition but this will lead to a less robust cascade control. Even with the existing tuning, the cascade PID control is unstable under the perturbed condition B; thus, an increase in controller aggressiveness will lead to smaller a smaller stability margin for the standard cascade PID control. The modified SP is stable under all of the perturbed conditions, but at the expense of larger IAE value than that of the CMSC. On average, the CMSC provides about $65 \%$ performance improvement over the modified SP scheme.

The closed-loop responses of the 3 different cascade control strategies at a perturbed condition are shown in Fig. 9. Notice that, under the perturbed condition the standard cascade PID control is unstable. Under the same perturbed condition, the CMSC scheme is not only stable but also shows smooth and fast responses to setpoint and disturbance changes compared with the modified SP scheme. Fig. 10 displays the plot of gain margin versus delay error for the CMSC and cascade PID control schemes. The gain margin for the CMSC is higher than that of the cascade PID control. This shows that the CMSC is more robust against modeling error than the standard cascade PID scheme.

\section{Conclusions}

In this paper, we have presented a generalized cascade multi-scale control (CMSC) scheme, which is an extension of the basic SISO MSC scheme recently reported in [22-23]. The proposed 
CMSC scheme can be applied to both self-regulating and non-self-regulating (integrating or unstable) processes with long time-delays. Furthermore, the CMSC structure can be reduced to an equivalent standard cascade PID control structure. The overall (master or slave) MSC controller can be easily put into the form of a PID or P controller augmented with a filter. Thus, the CMSC structure provides an attractive way to design an augmented filter together with a PID controller. Our numerical studies have shown that the use of this PID controller augmented with a first- or second-order filter in the primary-loop provides improved nominal performance and performance robustness over the PID controller without filter (including PID with a simple lag filter). Also it should be noted that, the proposed CMSC scheme is simpler to design and implement than some modified Smith predictor-based cascade control strategies, which are not often reducible to a standard cascade PID control structure. In future works, we will address: (1) the extension of the current CMSC scheme to parallel cascade processes, (2) autotuning procedure of the CMSC scheme for nonlinear processes, and (3) rigorous robustness and stability properties of the CMSC scheme.

\section{References}

[1] R. G. Franks, C. W. Worley, Quantitative analysis of cascade control, Ind. Eng. Chem., 6 (1956) 1074-1079.

[2] D. E. Seborg, T. F. Edgar, D. A. Mellichamp, Process Dynamics and Control, John Wiley \& Sons, 2004.

[3] B. A. Ogunnaike, W. H. Ray, Process Dynamics, Modeling and Control, Oxford University Press, 1994.

[4] F. G. Shinskey, Process Control Systems, McGraw-Hill, 1988. 
[5] J. R. Richards, J. P. Congalidis, Measurement and control of polymerization reactors, Comput. Chem. Eng., 30 (2006) 1447-1463.

[6] H. P. Huang, I. L. Chien, Y. C. Lee, Simple method for tuning cascade control systems, Chem. Eng. Commun.,165 (1998)89-121.

[7] Y. H. Lee, S. W. Park, M. Y. Lee, PID controller tuning to obtain desired closed loop responses for cascade control systems, Ind. Eng. Chem. Res., 37(1998) 1859-1865.

[8] K. K. Tan, T. H. Lee, R. Ferdous, Simultaneous online automatic tuning of cascade control for open loop stable processes, ISA T., 39 (2000) 233-242.

[9] T. Liu, D. Y. Gu, W. D. Zhang, Decoupling two-degree-of-freedom control strategy for cascade control systems, J. Process Contr., 15 (2005) 159-167.

[10] J. C. Jeng, M. W. Lee, Simultaneous automatic tuning of cascade control systems for closed-loop step response data, J. Process Contr., 22 (2012) 1020-1033.

[11] O. J. M. Smith, Closer control of loops with dead-time, Chem. Eng. Prog., 53 (1957) 217219.

[12] K. J. Astrom, C. C. Hang, B. C. Lim, A new Smith predictor for controlling a process with an integrator and long dead-time, IEEE T. Automat. Contr., 39 (1994) 343-345.

[13] S. Uma, M. Chidambaram, A. S. Rao, Set point weighted modified Smith predictor with PID filter controller for non-minimum phase (NMP) integrating processes, Chem. Eng. Res. Des., 88 (2010) 592-601. 
[14] A. M. de Paor, A modified smith predictor and controller for unstable processes with time delay, Int. J. Control, 41 (1985) 1025-1036.

[15] P. Garcia, T. Santos, J. E. Normey-Rico, P. Albertos, Smith predictor-based control schemes for dead-time unstable cascade processes, Ind. Eng. Chem. Res., 49 (2010)1147111481.

[16] D. G. Padhan, S. Majhi, Modified Smith predictor based cascade control of unstable time delay processes, ISA T., 51 (2012) 95-104.

[17] S. Uma, M. Chidambaram, A. S. Rao, Enhanced control of unstable cascade processes with time delays using a modified smith predictor, Ind. Eng. Chem. Res., 48 (2009) 3098-3111.

[18] S. Uma, M. Chidambaram, A. S. Rao, C. K. Yoo, Enhanced control of integrating cascade processes with time delays using modified smith predictor, Chem. Eng. Sci., 65 (2010) 1065-1075.

[19] D. G. Padhan, S. Majhi, Enhanced cascade control for a class of integrating processes with time delay, ISA T., 52 (2013) 45-55.

[20] T. Liu, W. Zhang, D. Gu, IMC based control strategy for open loop unstable cascade processes, Ind. Eng. Chem. Res., 44 (2005) 900-909.

[21] I. Kaya, P. Atherton, Use of Smith predictor in the outer loop for cascaded control of unstable and integrating processes, Ind. Eng. Chem. Res., 47 (2008) 1981-1987.

[22] J. Nandong, Z. Zang, Novel multiscale control scheme for nonminimum-phase processes, Ind. Eng. Chem. Res., 52 (2013) 8248-8259. 
[23] J. Nandong, Z. Zang, High-performance multi-scale control for stable, integrating and unstable time-delay processes, J. Process Contr., 23 (2013) 1333-1343.

[24] C. A. Desoer, M. Vidyasagar, Feedback Systems: Input-Output Properties, Academic Press, 1975. 
Table 1

The IAE values for CMSC and cascade PID schemes at the nominal and perturbed conditions for Example 1

\begin{tabular}{lcc}
\hline Condition & Proposed CMSC & Cascade PID \\
\hline Nominal & 37.6 & 46.1 \\
Case A & 53.7 & 62.4 \\
Case B & 46.6 & 64.7 \\
Case C & 39.3 & 54.1 \\
Case D & 41.7 & 49.2 \\
\hline
\end{tabular}

Table 2

The IAE values for CMSC, cascade PID and modified Smith predictor (SP) schemes at the nominal and perturbed conditions for Example 2

\begin{tabular}{lccc}
\hline Condition & Proposed CMSC & Cascade PID & Modified SP [17] \\
\hline Nominal & 11.1 & 13.2 & 14.7 \\
Case A & 11.3 & 11.8 & 13.8 \\
Case B & 14.9 & 18.6 & 19.6 \\
Case C & 11.2 & 16.4 & 15.9 \\
Case D & 13.7 & 20.4 & 19.4 \\
\hline
\end{tabular}

Table 3

The IAE values for CMSC, cascade PID and modified Smith predictor (SP) schemes at the nominal and perturbed conditions for Example 3

\begin{tabular}{lccc}
\hline Condition & Proposed CMSC & Cascade PID & Modified SP [16] \\
\hline Nominal & 28.2 & 30.0 & 37.5 \\
Case A & 28.7 & 40.1 & 68.8 \\
Case B & 36.8 & unstable & 68.1 \\
Case C & 37.4 & 40.8 & 46.3 \\
Case D & 45.3 & 47.5 & 70.5 \\
\hline
\end{tabular}


Table 4

PID controller settings based on CMSC, standard cascade and modified SP schemes for examples 1,2 and 3

\section{Example 1}

CMSC

Scheme

$$
G_{c 1}(s)=0.077\left(\frac{6 s^{2}+1.1 s+1}{s}\right) F(s) ; \quad F(s)=\frac{3.27 s^{2}+3.24 s+1}{2.74 s^{2}+2 s+1} ; \quad G_{c 2}(s)=1.22\left(\frac{2 s+1}{0.45 s+1}\right)
$$

Standard

Cascade

PID

$$
G_{c 1}(s)=0.035\left(\frac{5 s^{2}+0.9 s+1}{s}\right) F(s) ; \quad F(s)=\frac{1}{0.1 s+1} ; \quad G_{c 2}(s)=0.25\left(\frac{4 s+1}{s}\right)
$$

\section{Example 2}

CMSC

Scheme

$$
G_{c 1}(s)=0.41\left(\frac{21.7 s^{2}+8.33 s+1}{s}\right) F(s) ; \quad F(s)=\frac{0.065 s^{2}+0.47 s+1}{0.055 s^{2}+0.35 s+1} ; \quad G_{c 2}(s)=2.8\left(\frac{0.15 s+1}{0.072 s+1}\right)
$$

Standard

Cascade

PID

$$
G_{c 1}(s)=0.056\left(\frac{15.7 s^{2}+3.14 s+1}{s}\right) ; G_{c 2}(s)=1.4\left(\frac{2 s+1}{s}\right)
$$

${ }^{\$}$ Modified SP [17]

$$
\begin{aligned}
& G_{c s}(s)=0.5\left(1+\frac{1}{5 s s}+2 s\right) F_{1}(s) ; \quad F_{1}(s)=\frac{s+1}{0.1 s^{2}+0.5 s+1} ; \quad G_{f}(s)=\frac{1}{5 s+1} \\
& G_{c d}(s)=(0.0789+0.434 s) F_{2}(s) ; \quad F_{2}(s)=\frac{0.0833 s^{3}+0.583 s 2+1.5 s+1}{0.0789 s^{3}+0.395 s^{2}+0.753 s+1} ; \quad G_{c 2}(s)=\frac{s+1}{0.5 s+1}
\end{aligned}
$$

\section{Example 3}

CMSC

Scheme

$$
G_{c 1}(s)=0.11\left(\frac{39 s^{2}+30 s+1}{s}\right) F(s)
$$

$$
F(s)=\frac{3 s^{2}+2.68 s+1}{2.26 s^{2}+1.76 s+1} ; \quad G_{c 2}(s)=4.07\left(\frac{s+1}{0.1 s+1}\right)
$$

Standard

Cascade

$$
G_{c 1}(s)=0.088\left(\frac{25 s^{2}+30 s+1}{s}\right) ; \quad G_{c 2}(s)=0.51\left(\frac{10 s+1}{s}\right)
$$

${ }^{\$}$ Modified SP [17]

$$
\begin{aligned}
& G_{c s}(s)=\left(4.657+\frac{0.183}{s}+12.29 s\right) F_{1}(s) ; \quad F_{1}(s)=\frac{1}{2.86 s+1} ; G_{f}(s)=\frac{1}{36 s+1} \\
& G_{c d}(s)=\left(3.119+\frac{0.0921}{s}+6.616 s\right) F_{2}(s) ; \quad F_{2}(s)=\frac{3 s+1}{0.144 s+1} ; G_{c 2}(s)=0.5\left(\frac{20 s+1}{2 s+1}\right)
\end{aligned}
$$

\footnotetext{
${ }^{\$}$ The controller notations for the modified SP are as in Uma et al. [17].
} 


\section{Figure Caption}

Fig. 1. Conventional two-level cascade control strategy

Fig. 2. Block diagram of a 2-layer multi-scale control (MSC) scheme for a SISO process

Fig. 3. Block diagram of 2-level/2-layer cascade MSC (CMSC) scheme for cascade process

Fig. 4. Generalized 2-level CMSC scheme: (a) secondary, and (b) primary MSC systems

Fig. 5. Response under a perturbed condition $\left(\Delta_{1}=0.2, \Delta_{2}=-0.4, \delta_{1}=0.5, \delta_{2}=0.4\right)$ for Example 1

Fig. 6. Gain margin versus delay error for Example 1: (a) secondary- and (b) primary-loops under $\Delta_{1}=\Delta_{2}=0 ;$ (c) secondary- and (d) primary-loops under $\Delta_{1}=\Delta_{2}=0.2$

Fig. 7. Response under a perturbed condition $\left(\Delta_{1}=-0.4, \Delta_{2}=0.3, \delta_{1}=0.3, \delta_{2}=0.4\right)$ for Example 2

Fig. 8. Gain margin versus delay error for Example 2: (a) secondary- and (b) primary-loops under $\Delta_{1}=\Delta_{2}=0$; (c) secondary- and (d) primary-loops under $\Delta_{1}=\Delta_{2}=0.2$

Fig. 9. Response under a perturbed condition $\left(\Delta_{1}=0.25, \Delta_{2}=-0.2, \delta_{1}=0.1, \delta_{2}=0.1\right)$ for Example 3

Fig. 10. Gain margin versus delay error for Example 3: (a) secondary- and (b) primary-loops under $\Delta_{1}=\Delta_{2}=0 ;$ (c) secondary- and (d) primary-loops under $\Delta_{1}=\Delta_{2}=0.2$ 


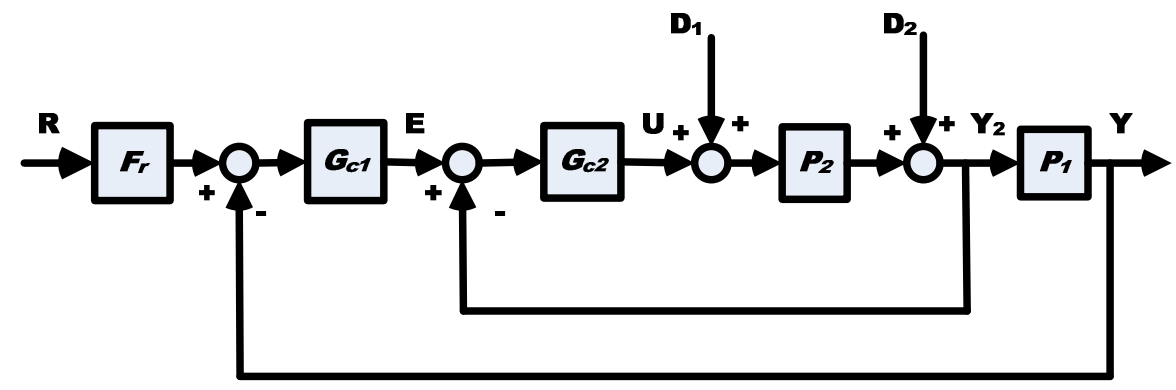

Figure 1 

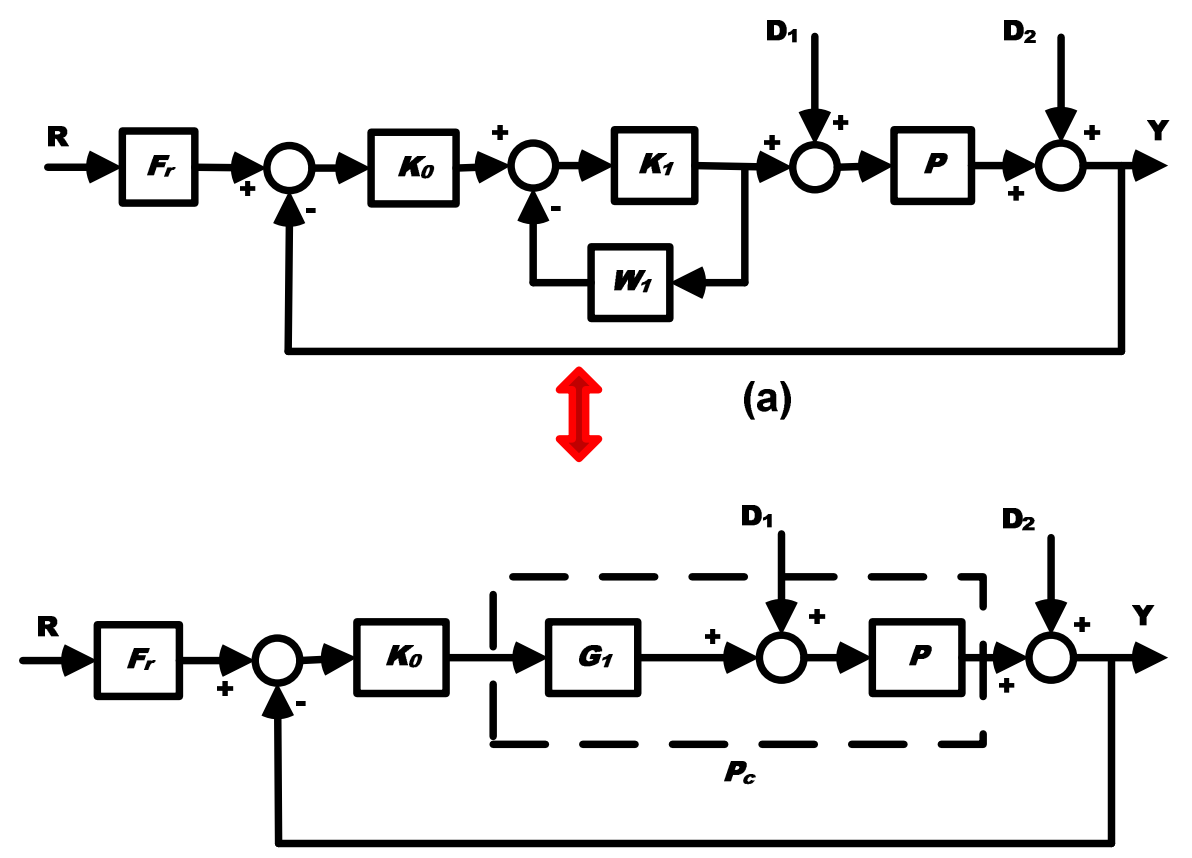

(b)

Figure 2 


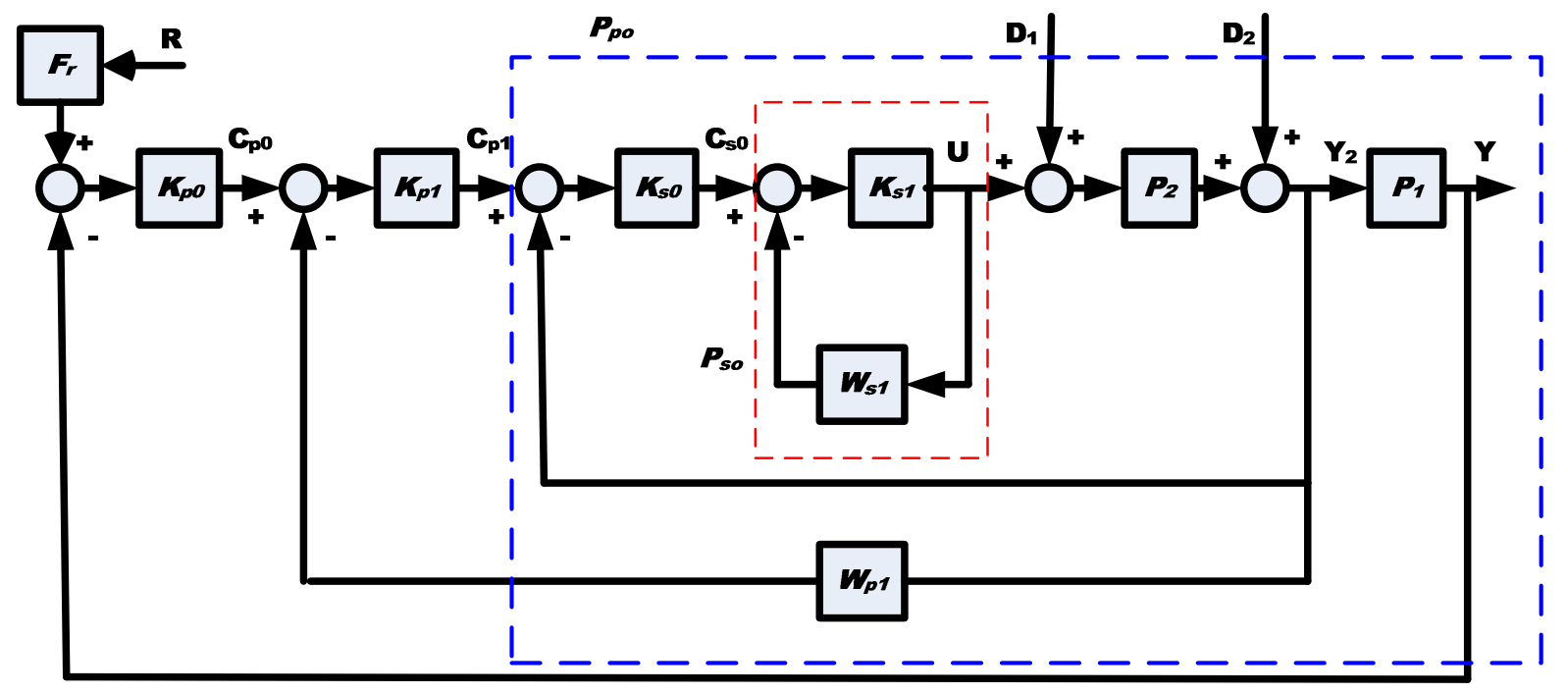

Figure 3

32 


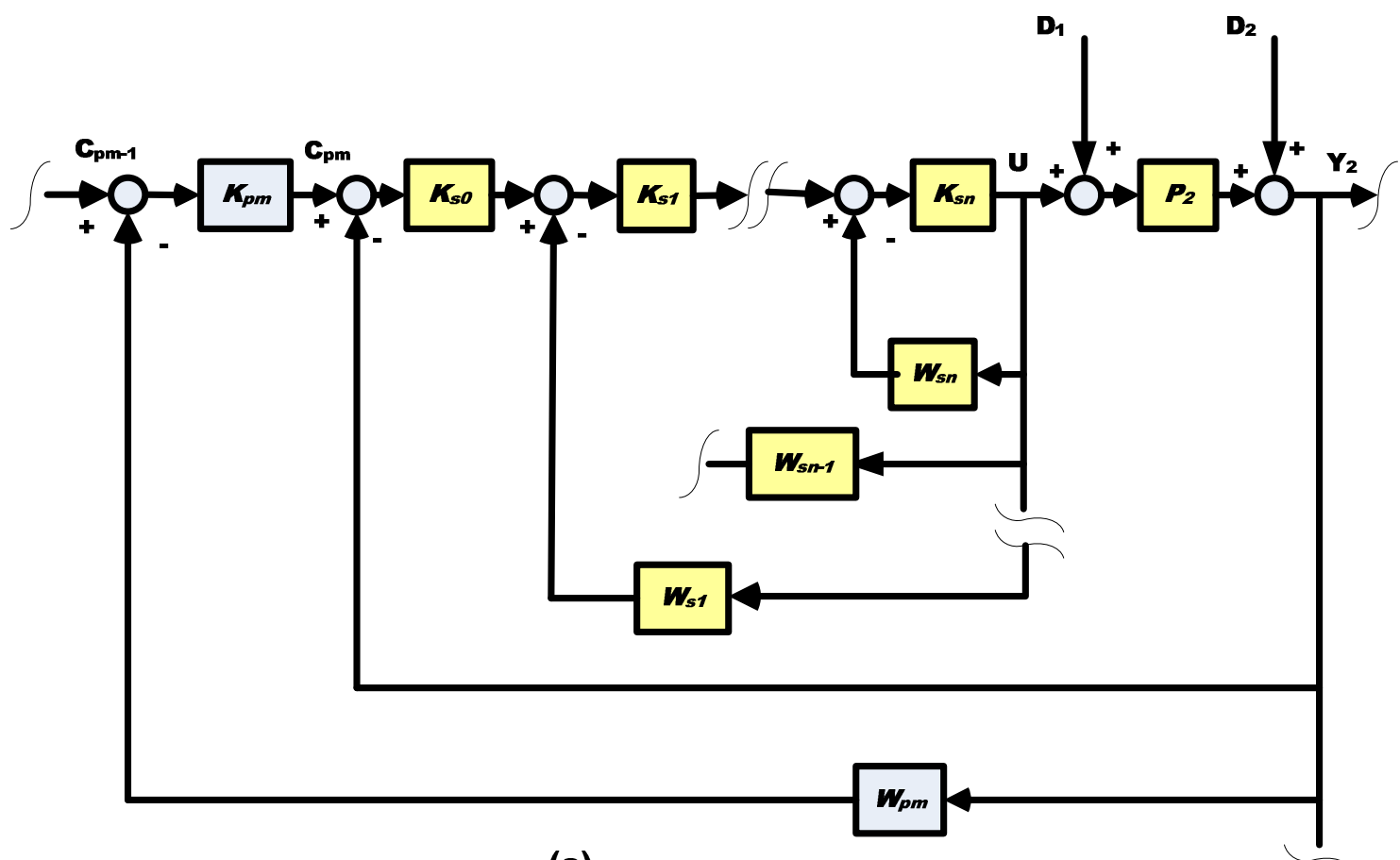

(a)

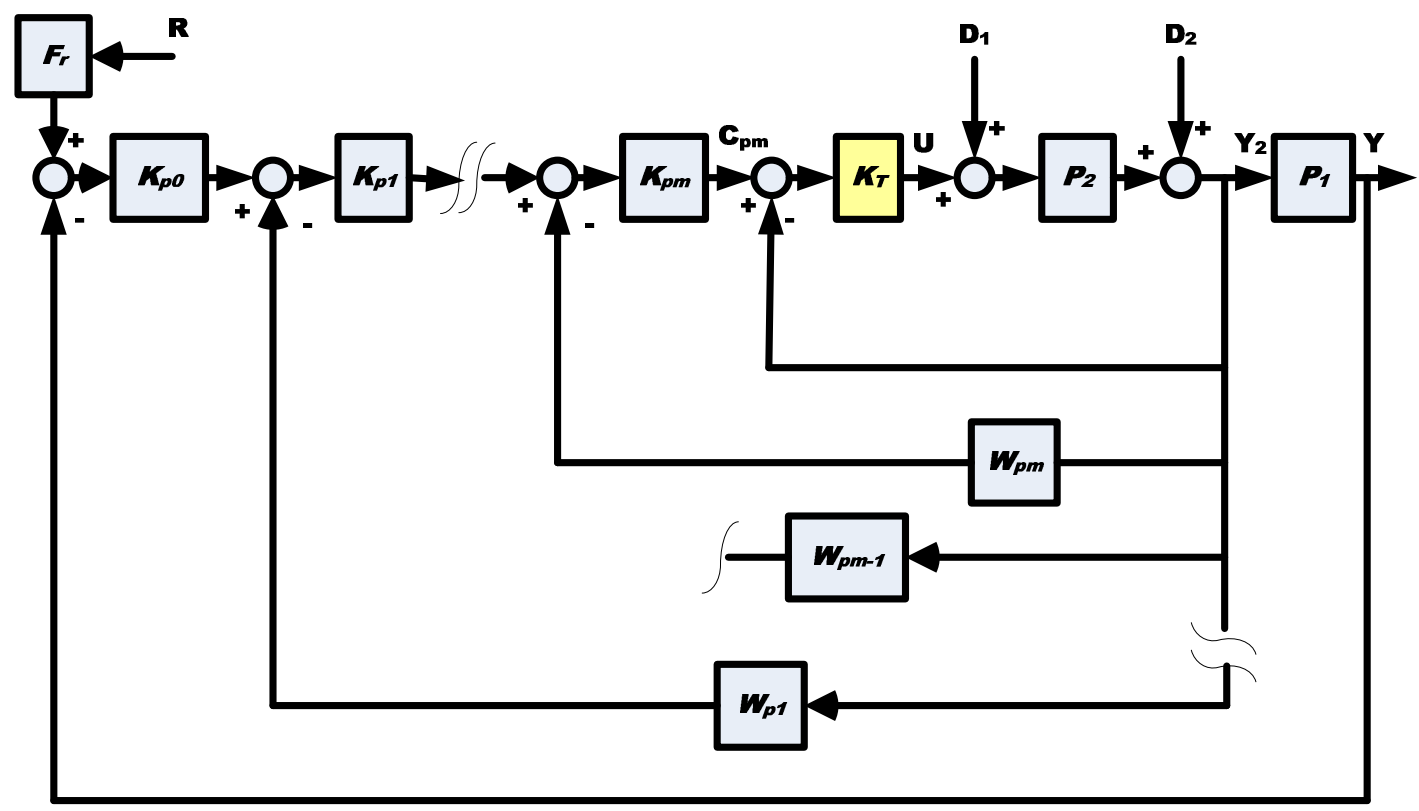

(b)

Figure 4 


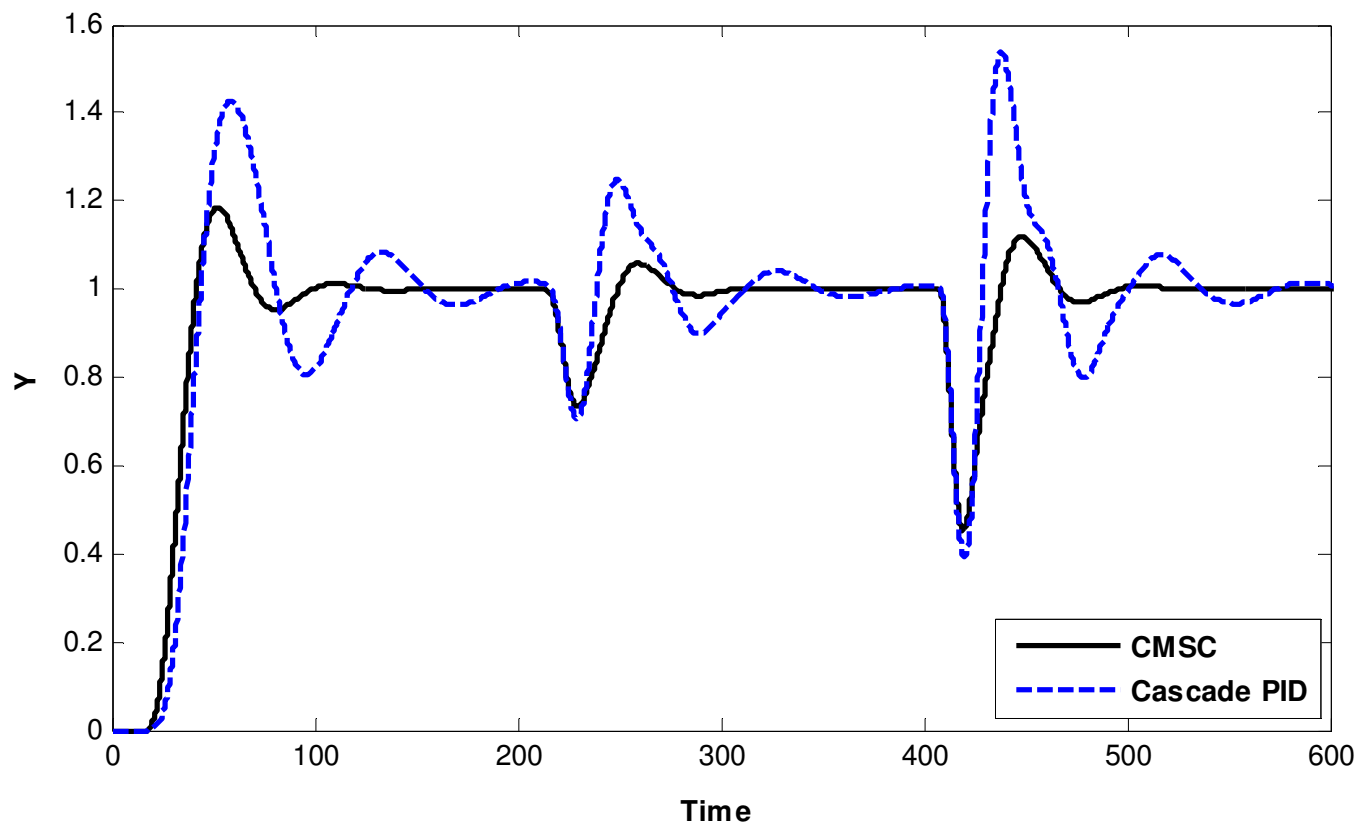

Figure 5 
(a)

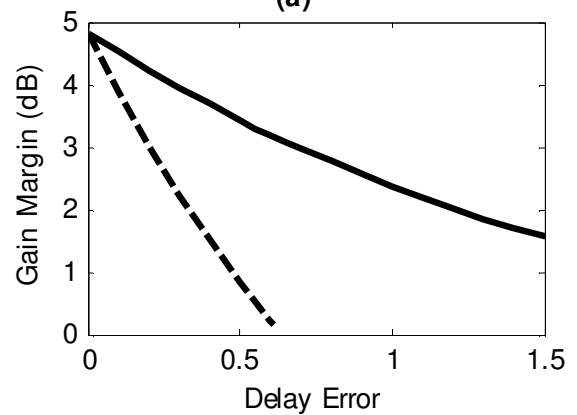

(c)

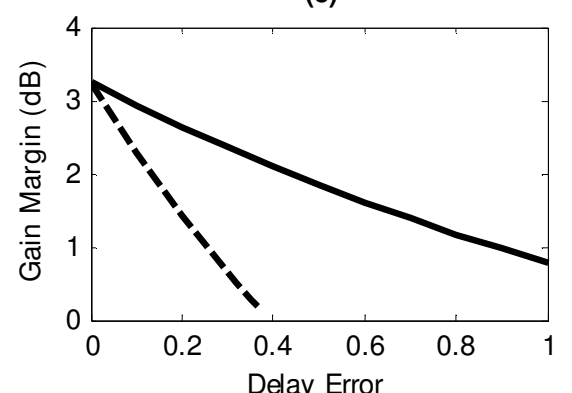

(b)

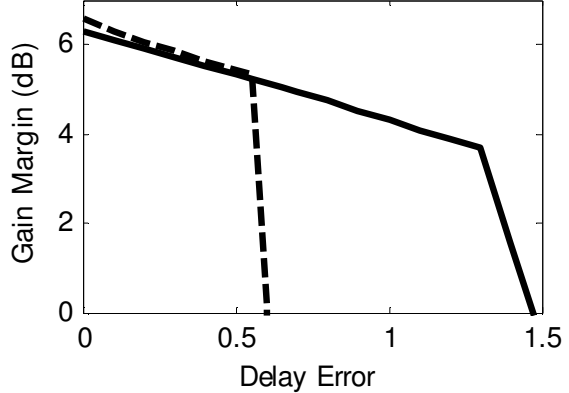

(d)

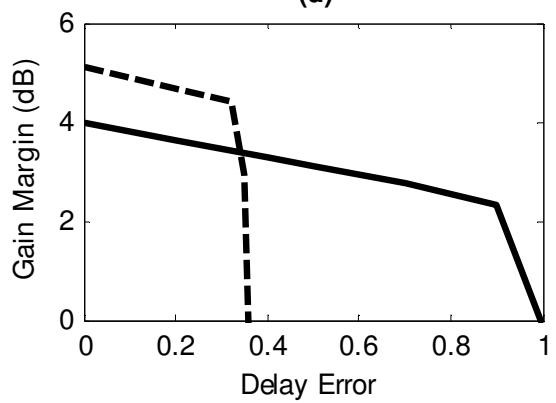

Figure 6 


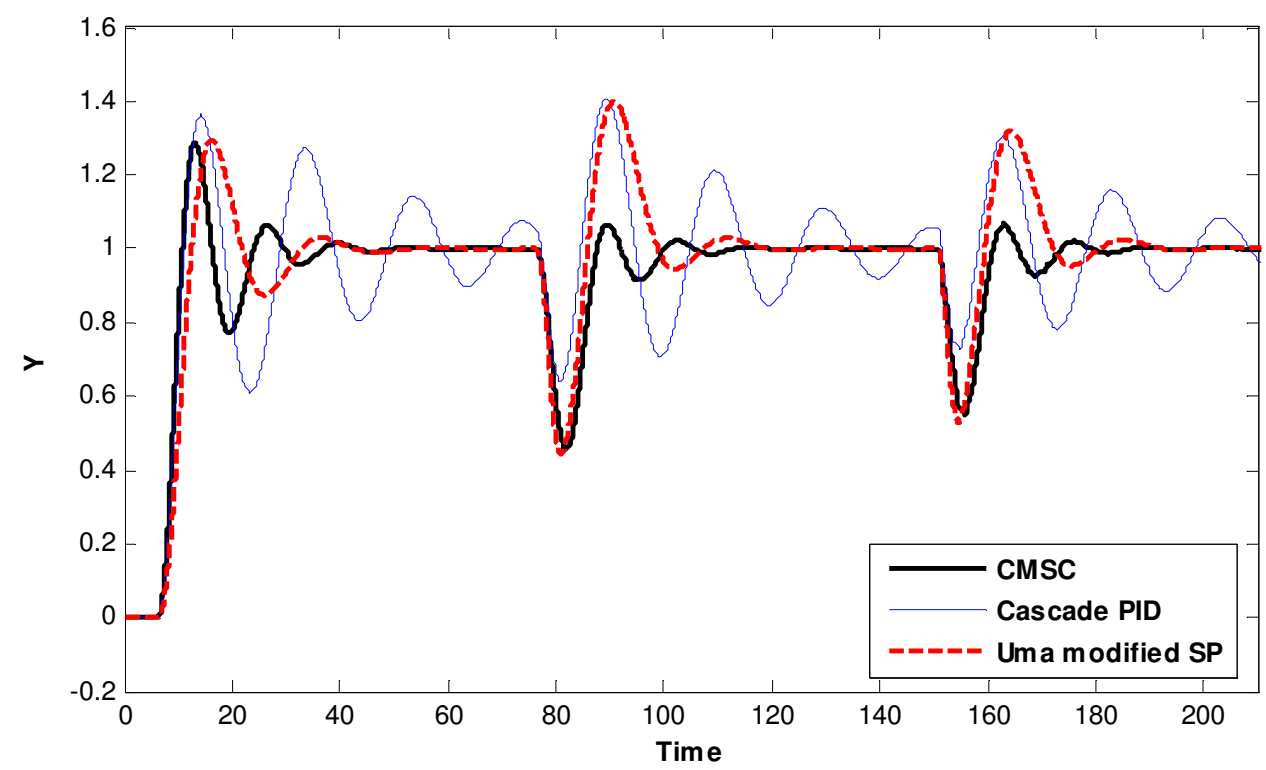

Figure 7 
(a)

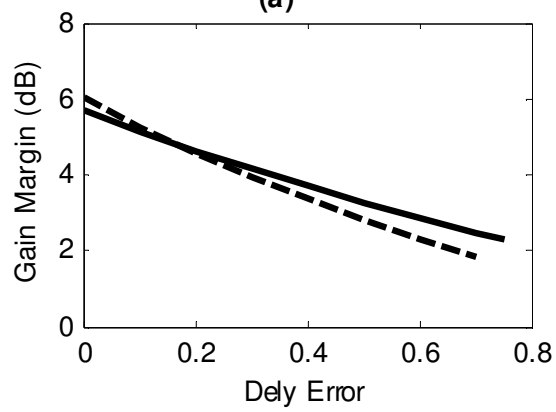

(c)

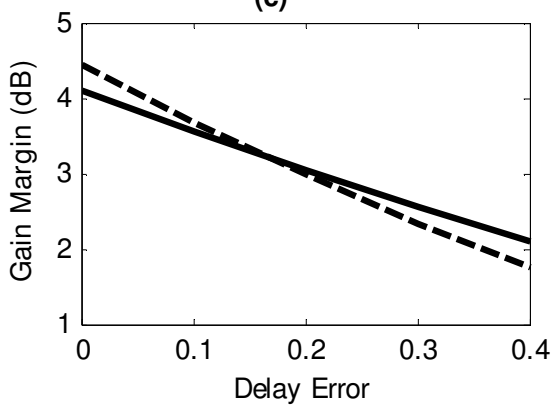

(b)

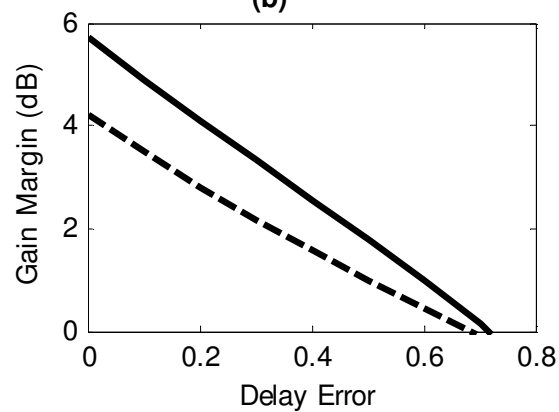

(d)

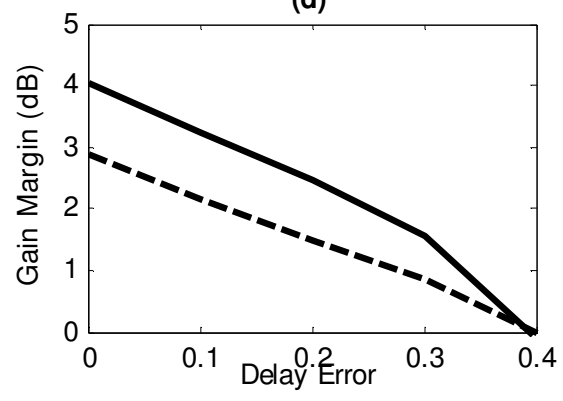

CMSC ヘーー・ Cascade PID

Figure 8 


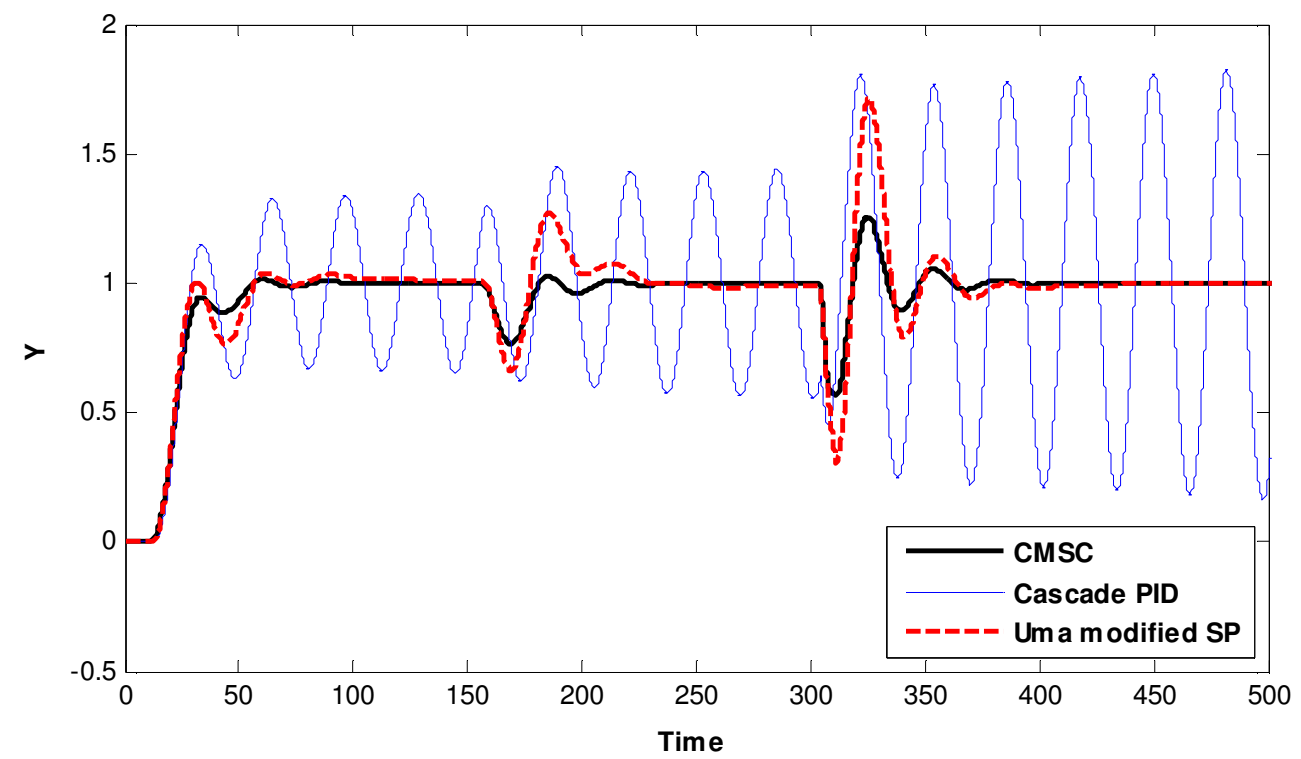

Figure 9 
(a)

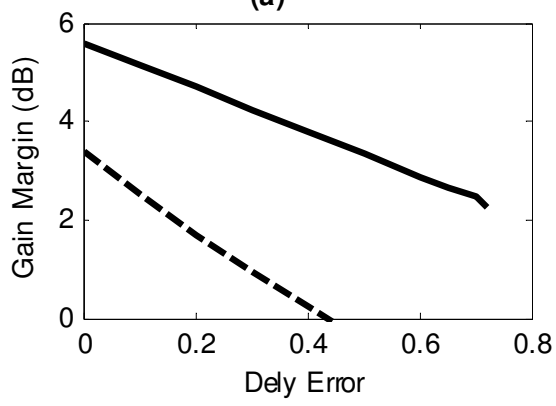

(c)

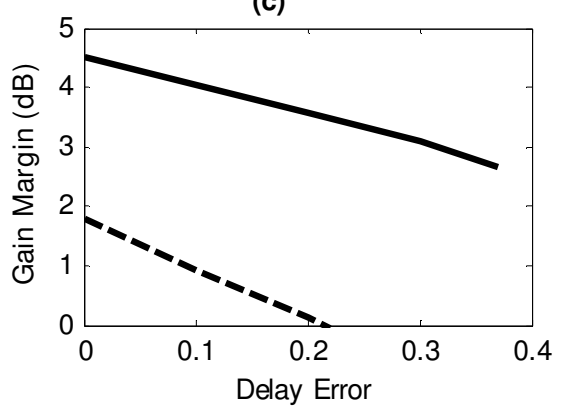

(b)

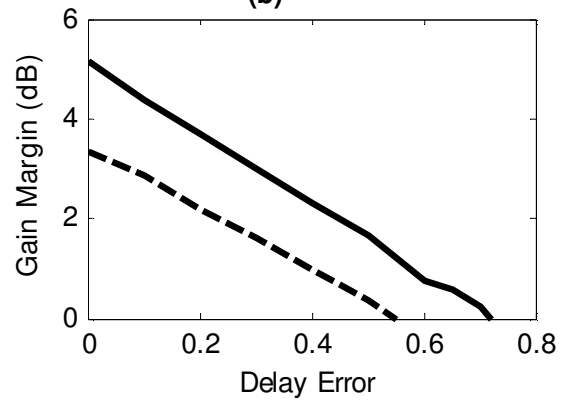

(d)

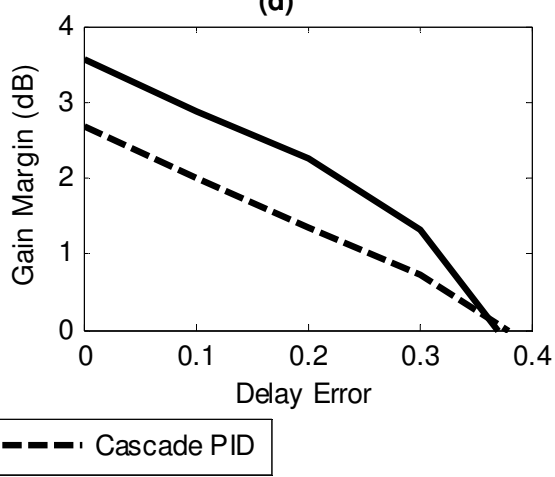

Figure 10 